\title{
Impact of cannabis on the neurocognitive performance of Jamaican adolescents
}

\author{
Karyl Powell-Booth ${ }^{1}$, W De La Haye ${ }^{2}$ and Samantha Longman-Mills ${ }^{2}$ \\ ${ }^{1}$ University of Technology, Kingston, Jamaica \\ ${ }^{2}$ The University of the West Indies, Mona Campus, Kingston, Jamaica
}

\begin{abstract}
Background: Cannabis is the most widely used illicit drug among Jamaican adolescent males. Neurocognitive impairment may be a residual effect of cannabis use. This study investigated the neuropsychological performances of adolescent male cannabis users on tests of learning, attention and memory.

Methods: Purposive sampling was utilized to recruit cannabis users $(n=30)$, while random sampling was utilized to select the control group of non-cannabis users $(n=32)$. Tests of learning, attention and memory were administered from the Wide Range Assessment of Memory \& Learning (WRAML 3) and the Wechsler Intelligence Scale for Children (WISC-IV). The t-test statistic was used to identify differences between groups.

Results: The mean age of the sample was $14.97 \pm 1.36$ years. There was a significant difference between the performance of cannabis users and non-users on all tests of learning, attention and memory conducted in this study. The largest mean difference was for Verbal Intelligence Quotient $($ VIQ), $t(60)=6.764, p<0.05$, followed by Digit Span Forward, $t(60)=16.954, p<0.05$ and Digit Span Backward, $t(60)=17.755, p<0.05$, while the smallest mean difference was for the Picture Memory Immediate subtest, $t(60)=4.638, p<0.05$.
\end{abstract}

Conclusion: Findings support the fact that cannabis use impairs learning, attention and memory. These results support the need for public health policies aimed at targeting early prevention strategies, demand reduction and the identification and treatment of adolescent cannabis users in Jamaica.

\section{Introduction}

Within Jamaica there is a cultural belief that cannabis use is associated with enhanced creativity, improved concentration [1] and even improved reflexes [2]. These mythical beliefs have resulted in high rates of cannabis use, particularly among the youth, despite cannabis use being illegal in Jamaica. A 1987 survey of patterns of substance misuse among post primary Jamaican students identified a $19.8 \%$ lifetime prevalence for cannabis use, while a 2000 Jamaican National School's Survey found the lifetime prevalence to have increased to $26.9 \%$ [3].

Research findings have suggested that cannabis use may impair neuro-cognitive functioning [4-6]. However, some researchers have suggested that the residual effects of heavy cannabis use on cognitive functions are reversible, lasting only a few days after cessation [7]. Results from one longitudinal study found that cannabis use does not have a long-term negative impact on intelligence [9], while others have found that heavy cannabis users had memory and learning impairments even after six weeks of supervised abstention [8].

There is a paucity of research on cannabis and neuro-cognitive performance in the Caribbean Region, including Jamaica. Given the widespread use of cannabis and its easy availability for Jamaican adolescents, it is important to identify if there are any neuro-cognitive effects associated with cannabis use, among the youth population. This study therefore investigates whether cannabis use among Jamaican adolescent males will result in lowered performances on neurocognitive tasks.

\section{Method}

A cross-sectional design was utilised to investigate the performance of adolescent male cannabis users on tests of verbal intelligence, language, learning, attention, concentration and memory as compared to non-cannabis users. Purposive sampling techniques were utilised to identify 35 adolescent male cannabis users between the ages of 13-17 years whose only drug of choice for the past 12 months was cannabis. These males reported smoking cannabis at least three times per week. Random sampling techniques were used to select a comparison group of 35 non-cannabis using adolescent males between the ages of 13-17 years, from two Secondary High Schools in Kingston, Jamaica. The mean age for the total sample was $14.97 \pm 1.36$ years. Participants in the cannabis using group who reported a history of a psychiatric diagnosis, or the use of any drug other than cannabis were excluded from the study. In the comparison group (non-cannabis users) participants who reported cannabis use within the last 12 months, or tested positive for metabolites of cannabis in their urine, were excluded from the study.

Cannabis users were required to abstain from using for a period

Correspondence to: Winston De La Haye, M.D., M.P.H., D.M, Lecturer in Psychiatry, Department of Community Health and Psychiatry, The University of the West Indies, Mona Campus, Kingston, Tel: +1 (876) 383-0348; Fax: +1 (876) 631-3742; Intl. voice mail: +1 (514) 800-2627; E-mail: wdela@yahoo.com

Key words: cannabis, memory, adolescent, Jamaican and male

Received: October 04, 2016; Accepted: October 21, 2016; Published: October 26, 2016 
of 24 - 48 hours prior to participating in the testing process. Of the 35 participants initially recruited for the cannabis use group, 3 were expelled from school and 2 chose to withdraw from the study. Of the 35 participants in the non-user control group, 3 were excluded from the study because their urine contained metabolites of cannabis. A total of 30 cannabis users and 32 non-users were inter viewed for the study.

\section{Measures}

The measures utilised in this study are the: [1] Wide Range Achievement Test, Third Edition (WRAT 3); [2] subtests from the Wide Range Assessment of Memory and Learning (WRAML) and [3] subtests from the Wechsler Intelligence Scales for Children, Fourth Edition (WISC-IV). The WRAT 3 is a widely used screening instrument for the presence of academic difficulties. It is a standardised instrument that has been normed for use with individuals between the ages $5-75$ years, assessing the basic skills for reading, spelling and math computation. Participants were screened for academic difficulties with the WRAT 3 and those identified were excluded from the study.

The WRAML is a standardized neuropsychological instrument for the assessment of learning and memory. The Picture Memory, Verbal Learning and Story Memory subtests were selected to measure visual and verbal memory, as well as auditory sequencing and attention to details. The WRAML has been found to have reliability coefficients of between .80 and .85 .

The WISC-IV is a standardized benchmark instrument used to measure the intelligence of children and adolescents. The Digit Span, Coding, Vocabulary, and Similarities subtests of the WISCIV, were selected to assess working memory, mental manipulation, concentration, attention, language development, general verbal intelligence, educational background and overall verbal comprehension.

\section{Procedure}

Ethical approval was obtained from The University of the West Indies, Faculty of Medical Sciences' Ethics Committee and informed consent was obtained from the guardians of each volunteering student. The students also signed an assent form prior to participating in the study. Participants from both groups (users and non-users of cannabis) were asked to complete a self-administered demographic questionnaire reporting their age, class grade, and substance use history. Participants in the non-user group were tested for the presence of cannabis metabolites in their urine. All participants were screened with the WRAT 3 and those with identified academic difficulties were excluded from the study.

The WRAML and WISC-IV subtests were then administered to the selected students to assess their neurocognitive functions. Data analysis was accomplished using the Statistical Package for the Social Sciences version 14 (SPSS v.14) and t-tests were conducted to assess if there were any significant differences between the performances of cannabis users and non-users.

\section{Results}

The sample consisted of 62 male students, 30 cannabis users and 32 non-users. The mean age of the total sample was $14.97 \pm 1.36$ years, with the mean age for users being $15.73 \pm 1.173$ years and non-users $14.25 \pm 1.14$ years. Approximately $18 \%$ of cannabis users started smoking cannabis before age 13 , with a reported mean age of initiation being 13.4 years. Seventy eight percent $(78 \%)$ of users indicated that they smoked at parties/clubs, and with friends, as opposed to smoking by themselves; with smoking occurring mostly on the weekend, with Saturday being reported as the most popular day of the week to smoke cannabis.

Significant mean differences were observed between the neuropsychological performances of cannabis users versus non-users (Table 1). The largest mean differences were observed for Verbal Intelligence Quotient (VIQ), $t(60)=6.764, p<0.05$, followed by Digit Span Forward, $t(60)=16.954, p<0.05$ and Digit Span Backward, $t$ $(60)=17.755, p<0.05$, while the smallest mean difference was for the Picture Memory Immediate subtest, $t(60)=4.638, p<0.05$ (Table 1 ). Nonusers had better neuro-cognitive performances than cannabis users on the Picture Memory, Verbal Learning and Story Memory subtests, both immediate and delayed. However, the decline in performance was more evident among the users than the non-users, on the delayed tests (Table 2).

\section{Discussion}

The mean age of cannabis initiation in this study was found to be early adolescence, as seen in other Caribbean studies $[3,11]$. As adolescence is the developmental period for experimentation and risky behaviours, along with the cultural acceptability of cannabis use in Jamaica, this finding was anticipated. However, cannabis use during adolescence is a cause for serious concern as the adolescent brain is still undergoing neural development and may be susceptible to impairments in neuro-cognitive functioning.

Cannabis users exhibited lower scores on all assessed neuropsychological functions as compared to non-users. However, the greatest mean differences were observed through significantly lowered Verbal Comprehension as well as Digit Span scores. This finding implicates cannabis use during adolescence with impairing the neurocognitive functions of working memory, attention, concentration, mental manipulation, language development and verbal intelligence. Cannabis users also had significantly lower visual, verbal and working memory scores than those of non-cannabis users with the largest differences being seen on the delayed subtests. The observance of significantly lower scores on the delayed subtests implies that the longterm memory of cannabis users may be more susceptibility to neurocognitive decline.

Cannabis users had lower scores on all tests of learning, attention and memory than non-users. This is consistent with findings from previous research studies on the impact of cannabis on neuropsychological performance [13-18]. A meta-analytic study by

Table 1. Mean differences in subtest scores between cannabis users and non-users.

\begin{tabular}{|c|c|c|c|c|}
\hline \multicolumn{1}{|c|}{ Sub-Tests } & t & df & p & $\begin{array}{c}\text { Mean } \\
\text { Difference }\end{array}$ \\
\hline WRAML & & & & \\
\hline Picture Memory Immediate & $4.638^{*}$ & 60 & 0.00 & 1.03958 \\
\hline Picture Memory Delayed & $6.785 *$ & 60 & 0.00 & 1.39792 \\
\hline Verbal Learning Immediate & $6.334 *$ & 60 & 0.00 & 1.74375 \\
\hline Verbal Learning Delayed & $7.686^{*}$ & 60 & 0.00 & 2.1500 \\
\hline Story Immediate & $8.062^{*}$ & 60 & 0.00 & 2.06875 \\
\hline Story Delayed & $7.864 *$ & 60 & 0.00 & 2.29792 \\
\hline WISC-IV & & & & \\
\hline Digit Span Forward & $16.954 *$ & 60 & 0.00 & 6.47083 \\
\hline Digit Span Backward & $17.754^{*}$ & 60 & 0.00 & 6.60417 \\
\hline Coding & $11.831 *$ & 60 & 0.00 & 3.18125 \\
\hline Vocabulary & $5.859 *$ & 60 & 0.00 & 1.54792 \\
\hline Similarities & $5.401 *$ & 60 & 0.00 & 1.32708 \\
\hline Verbal Comprehension Index & $6.764 *$ & 60 & 0.00 & 6.64792 \\
\hline$* p<.05$ & & & & \\
\hline
\end{tabular}


Table 2. Subtest scale scores for cannabis users and non-users

\begin{tabular}{|l|c|c|c|c|}
\hline Subtest scale scores & \multicolumn{2}{c|}{ Groups } \\
\hline & \multicolumn{2}{|c|}{$\begin{array}{c}\text { Non-User } \\
\mathrm{n}=32\end{array}$} & $\begin{array}{c}\text { Cannabis } \\
\text { User } \mathrm{n}=30\end{array}$ & \\
\hline & $\mathrm{M}$ & $\mathrm{SD}$ & $\mathrm{M}$ & $\mathrm{SD}$ \\
\hline Pic Memory Immediate & 12.41 & 0.91 & 11.37 & 0.85 \\
\hline Pic Memory Delayed & 11.53 & 0.8 & 10.13 & 0.82 \\
\hline Verbal Learning Immediate & 13.34 & 1.09 & 11.60 & 1.07 \\
\hline Verbal Learning Delayed & 12.25 & 1.14 & 10.10 & 1.06 \\
\hline Story Immediate & 13.47 & 1.13 & 11.40 & 0.85 \\
\hline Story Delayed & 12.53 & 1.34 & 10.23 & 0.89 \\
\hline Digit-span forward & 11.94 & 0.98 & 5.47 & 1.91 \\
\hline Digit-span backward & 10.94 & 0.76 & 4.33 & 1.84 \\
\hline Coding & 12.28 & 1.08 & 9.10 & 1.03 \\
\hline VIQ & 104.28 & 3.85 & 97.63 & 3.89 \\
\hline Vocabulary & 12.78 & 1.24 & 11.23 & 0.77 \\
\hline Similarities & 12.09 & 1.06 & 10.77 & 0.85 \\
\hline
\end{tabular}

Grant, et al. [19] also identified impairment in the ability of chronic users of cannabis to recall new information, though findings by Schwartz [20] and Lyons [21] indicate an absence of long-term residual effects of cannabis use on cognitive abilities.

Traditionally, Jamaicans view cannabis use as providing many benefits. These findings are an important step in providing empirical evidence for possible cognitive impairment from cannabis use, among the adolescent population. Further research is needed to determine dose-related, in addition to long-term residual effects of cannabis use on neuropsychological performance in the Caribbean. Understanding the relationship between the complex factors that influence neurocognitive performance of cannabis users should further help to inform the development of public policy and legislation in Jamaica and the Caribbean.

\section{Limitations}

The sample size of 30 for the user group even though deemed sufficient, was still small and the present study consisted of male participants only. It would be of interest to know if there is a gender difference in cannabis users' in performance on neurocognitive tests of memory.

\section{Conclusion}

The findings suggest that there is a significant difference in performance between Jamaican male adolescent cannabis users and non-users on neuro-cognitive tests. Users of cannabis displayed cognitive deficits on all tests of memory, intelligence, language and attention that were conducted. The present findings lend new support to the notion that cannabis use may impair neurocognitive functioning. There are implications for poor school performance by adolescent users of cannabis in Jamaica. These results support the need for public health policies aimed at targeting early prevention strategies, demand reduction, identification and treatment of adolescent cannabis users in Jamaica.

\section{References}

1. Chevannes B (1988) Background to Drug Use In Jamaica. The University of the West Indies Kingston, Jamaica: Institute of Social and Economic Research (ISER).

2. Rubin (1975) Cannabis and Culture. The Hague, Paris: Mouton Publishers.

3. Douglas K-G Patterns of (2000) Substance Use and Abuse Among Post Primary Students in Jamaica. Kingston: Planning Institue of Jamaica.

4. Kalechstein A, van Gorp WG (2011) Neuropsychology and Substance Use: State-of-
the-Art and Future Directions: Taylor \& Francis.

5. Medina KL, Hanson KL, Schweinsburg AD, Cohen-Zion M, Nagel BJ, et al. (2007) Neuropsychological functioning in adolescent marijuana users: Subtle deficits detectable after a month of abstinence. Journal of the International Neuropsychological Society 13: 807-820. [Crossref]

6. Kanayama G, Rogowska J, Pope HG, Gruber SA, Yurgelun-Todd DA (2004) Spatial working memory in heavy cannabis users: a functional magnetic resonance imaging study. Psychopharmacology 176: 239-247. [Crossref]

7. Pope HG Jr, Gruber AJ, Hudson JI, Huestis MA, Yurgelun-Todd D (2001) Neuropsychological performance in long-term cannabis users. Arch Gen Psychiatry 58: 909-915. [Crossref]

8. Schwartz RH, Gruenewald PJ, Klitzner M, Fedio P (1989) Short-term memory impairment in cannabis-dependent adolescents. American Journal of Diseases of Children 143: 1214-1219. [Crossref]

9. Fried P, Watkinson B, James D, Gray R (2002) Current and former marijuana use: preliminary findings of a longitudinal study of effects on IQ in young adults. CMAJ 166: 887-891.

10. Burton D, Donders J, Mittenberg W (1996) A structural equation analysis of the Wide Range Assessment of Memory and Learning (WRAML) in the standardization sample. Child Neuropsychology 2: 39-47.

11. De La Haye W, Harris J (2005) Early Age of Onset of Substance Abuse in Clients Treated in an Adolescent Substance Abuse Clinic in a General Hospital in Jamaica [Abstract]. West Indian Med J 5 (suppl 5): 24.

12. Plancherel B, Bolognini M, Stéphan P, Laget J, Chinet L, et al. (2005) Adolescents' beliefs about marijuana use: a comparison of regular users, past users and never/ occasional users. J Drug Educ 35: 131-146. [Crossref]

13. Pope HG Jr, Gruber AJ, Hudson JI, Huestis MA, Yurgelun-Todd D (2001) Neuropsychological performance in long-term cannabis users. Arch Gen Psychiatry 58: 909-915. [Crossref]

14. Kelleher LM, Stough C, Sergejew AA, Rolfe T (2004) The effects of cannabis on information-processing speed. Addict Behav 29: 1213-1219. [Crossref]

15. Pope HG Jr, Yurgelun-Todd D (1996) The Residual Cognitive Effects of Heavy Marijuana Use in College Students. JAMA 275: 521-527. [Crossref]

16. Mendhiratta SS, Varma VK, Dang R, Malhotra AK, Das K, et al. (1988) Cannabis and cognitive functions: a re-evaluation study. Br J Addict 83: 749-753. [Crossref]

17. Fried PA, Watkinson B, James D, Gray R (2002) Current and Former Marijuana Use: Preliminary Findings of a Longitudinal Study of Effects on IQ in Young Adults. Canadian Med Association J 166: 887-891.

18. Bolla KI, Eldreth DA, Matochik JA, Cadet JL (2005) Neural substrates of faulty decision-making in abstinent marijuana users. Neuroimage 26: 480-492. [Crossref]

19. Grant I, Gonzalez R, Carey CL, Natarajan L, Wolfson T (2003) Non-acute (Residual) Neurocognitive Effects of Cannabis Use: A Meta-analytic Study. $J$ Int Neuropsychol Soc 9: 679-689. [Crossref]

20. Schwartz RH, Gruenewald PJ, Klitzner M, Fedio P (1989) Short-term memory impairment in cannabis-dependent adolescents. Am J Dis Child 143: 1214-1219. [Crossref]

21. Lyons MJ, Bar JL, Panizzon MS, Toomey R, Eisen S, et al. (2004) Neuropsychological consequences of regular marijuana use: a twin study. Psychol Med 34: 1239-1250. [Crossref]

Copyright: (C2016 Powell-Booth K. This is an open-access article distributed under the terms of the Creative Commons Attribution License, which permits unrestricted use, distribution, and reproduction in any medium, provided the original author and source are credited. 